\title{
TEAM SYNTEGRITY AS A LEARNING TOOL: SOME CONSIDERATIONS ABOUT ITS CAPACITY TO PROMOTE CRITICAL LEARNING ${ }^{1}$
}

\author{
Andrés Mejía D. (PhD) \\ Departamento de Ingeniería Industrial \\ Universidad de Los Andes \\ Calle 19A No.1-37 Este \\ Bogota D.C. Colombia
}

Tel: (57 1) 3394949 ext.3099

Fax: (57 1) 3324321

E-mail: jmejia@uniandes.edu.co

\author{
Angela Espinosa (PhD) \\ Business School \\ The University of Hull \\ Scarborough Campus, Filey Rd., \\ Scarborough, YO11 3AZ NY, U.K. \\ Tel: (44 1723) 357260 \\ Fax (44 1723) 374265 \\ E-mail: a.espinosa@hull.ac.uk
}

\begin{abstract}
Team Syntegrity is a systemic protocol designed by Stafford Beer for organising nonhierarchical and democratic conversations among a set number of participants. Contrary to those of other systemic protocols or methodologies, the rules or restrictions it provides are about the conversational structure, but make no reference to conversational contents. As a study of Paulo Freire's idea of critical consciousness reveals, while structure-based restrictions can help to properly deal with the views already brought into the conversation by its participants, they cannot guarantee that all the relevant aspects or views will actually be brought in and considered. The specification of some aspects of Team Syntegrity, however, such as the participant selection, and the roles of advocates, critics, and facilitators, may still have some effect on the contents that are likely to be taken into account in the conversation. Therefore, they constitute possible sources for this protocol's future improvement.
\end{abstract}

Keywords: Critical learning, Team Syntegrity, conversational restrictions, critical consciousness, democracy.

\section{INTRODUCTION}

Team Syntegrity has resulted from the last eleven years of research of the late Professor Stafford Beer. Following his work on the Viable System Model, in which he introduced criteria for studying the capacity of social organisations for adaptation and survival, he tried to respond to some key questions related to the development of non-hierarchical, democratic organisations able to self-organize and work cooperatively when agreeing on strategic or conflictive issues. Some of the issues he addressed can be expressed in the following questions: How to design communication protocols that facilitate a participatory and fair dialog among people holding different viewpoints that are equally legitimate? How to promote conversational contexts that facilitate the building of agreements from a diversity of speeches? How to integrate distributed knowledge to develop shared knowledge as

\footnotetext{
${ }^{1}$ An earlier version of this paper was presented at the ISSS Conference, Crete, June 2003, and published in its proceedings.
} 
criteria for guiding social actions? (Beer, 1994). The result of Beer's work, Team Syntegrity, is a protocol designed to organise an event in which some topics are discussed by a certain number of participants. This protocol consists of a set of rules about the conversational interactions among the persons who participate in the event.

Now, while it is rather clear to us that the conversational protocol developed by Beer does overcome many of the barriers to genuine communication and participation that have traditionally affected processes of learning and of decision making, there is still the little-examined question of exactly how completely it does it, and along what dimensions. There is also the related question of to what extent Team Syntegrity promotes learning about the topics that are discussed, by the participants in events, in a critical manner, or critical learning. Our main purpose in this paper is, precisely, to examine these issues in depth.

Our concern with these questions was partly prompted by the reflections that followed a previous experience in which Team Syntegrity was used to coordinate the development of an academic postgraduate course on the subject of re-engineering (Espinosa, 2000). This experience left us with some questions about the effectiveness of the protocol for creating a truly democratic learning context. After reviewing the experience with students and facilitators, we concluded that even if the students felt the protocol as the most democratic learning context they had experienced before, not all of them acted as truly critical and autonomous learners. Drawing some inspiration from the work of Paulo Freire, in the process review it was considered that their learning process might be enhanced by a more critical awareness on the topics explored, developing themselves as what we have termed critical active learners; that is, as people able to responsibly develop their own awareness and critical stances about the social meanings guiding their purposes and actions (Freire, 1973; and Espinosa, 2000). Interestingly, this seems to suggest that such a democratic protocol could be particularly useful when the participants were already active and intellectually autonomous learners; but some limitations could arise if they were not. From our experience we have come to believe that Team Syntegrity is still helpful in many ways in respect of the creation of democratic learning contexts; but then can it be used in some way such that it more fully helps participants become critical autonomous learners?

In this paper we will not propose any addition or modification to the protocol, and instead will limit ourselves to the examination in depth of some aspects of Team Syntegrity that can help give an answer to the questions presented above. In order to do this, we will analyse conversational restrictions in general, by means of a comparison of those provided by Team Syntegrity with those provided by other systemic approaches. The emphasis here is put on those linguistic spaces that are opened, or closed, or simply left untouched, by the different kinds of conversational restrictions established by any methodology or protocol. When comparing them, a main distinction we will invoke will be that between restrictions on the form of the interactions, and interpretative restrictions on content. The ways in which both 
contribute to create a democratic learning context and help participants become critical learners, is the main focus of analysis.

We start with a brief explanation of Team Syntegrity in section 1. Then, in section 2, three other systemic approaches are introduced, and explored as regards the way they attempt to structure-and therefore restrict-various aspects of conversation. Section 3 is dedicated to exploring the ways in which those restrictions may or may not help promote critical learning depending on their nature. For this purpose, the Freirean notion of critical consciousness (Freire, 1970 and 1973) is introduced. Following that, in section 4 the analysis is focused, and some conclusions are drawn concerning Team Syntegrity in particular.

\section{TEAM SYNTEGRITY}

Team Syntegrity has been described as an approach that supports decision processes in a participative non-hierarchical organisation, by means of a conversational protocol based on the geometric structure of some polyhedra. The main source in the literature for the principles and the protocol of Team Syntegrity is still Beer's book (Beer, 1994). It has been said that there is an emancipatory purpose underlying Team Syntegrity (Jackson, 2000), manifested in its concern for the development of symmetrical conversational interactions between people, in such a way that power relations do not affect the outcomes negatively (decisions, learning, etc.) Now, the particular strategy that Stafford Beer uses is to design the structure of communication, by means of a protocol. The protocol is the set of rules that a group of people agree to respect, to develop a discussion of issues that are of interest to everybody, but about which each one may hold a different viewpoint. It seeks to produce a conversation in which every participant's voice is heard, and no single one of them is allowed to dominate over the others by means of authoritarian imposition. For this purpose, Beer uses polyhedra, whose regular structure can be assimilated to the fairness required for a democratic conversation.

The most used version of Team Syntegrity is the one based on the icosahedron, for thirty participants to discuss a general topic expressed in an opening question, although other versions using other polyhedra have also been developed (Beer, 1994). The icosahedron has twenty (20) faces, twelve (12) vertices, and thirty (30) edges. Each edge represents one participant, and the group of 30 participants is called the infoset. Each vertex corresponds to one subtopic of discussion. The set of twelve subtopics constitutes the general topic. As suggested by the fact that each edge links two vertices, each participant will be directly involved in the discussion of two subtopics as a discussant, or advocate. Consequently, participant $g-a$ in figure 1 is involved in two groups in the role of advocate $(G$ and $A$ ), in each with four other participants, defined by the geometry of the icosahedron (g-c, g-e, g-i, and g-k, for group $G$ ). The groups of advocates in the discussion of any subtopic are called teams, and therefore advocates are also called team members. Each team is responsible for the conclusions reached about its own subtopic. However, apart from this role, each 
person will also participate in the discussions of two more subtopics, in the role of critic. The critic is someone who helps the team members to become aware of issues not addressed or properly discussed, and thus helps the discussion become a more enriching one. They should act as "devil advocates", by challenging the team members to review their own agreements, questioning shared beliefs behind them. Additionally, each participant will witness the discussion of two other subtopics simply as an observer. The event organisers provide logistic support for the team meetings; however, responsibility for the content and conclusions produced by each working group belongs to the group itself. As part of this support, there are facilitators, whose role is to make sure all advocates and critics have fair opportunities to participate, and to summarise the conversation, but not to influence the content of their contributions.

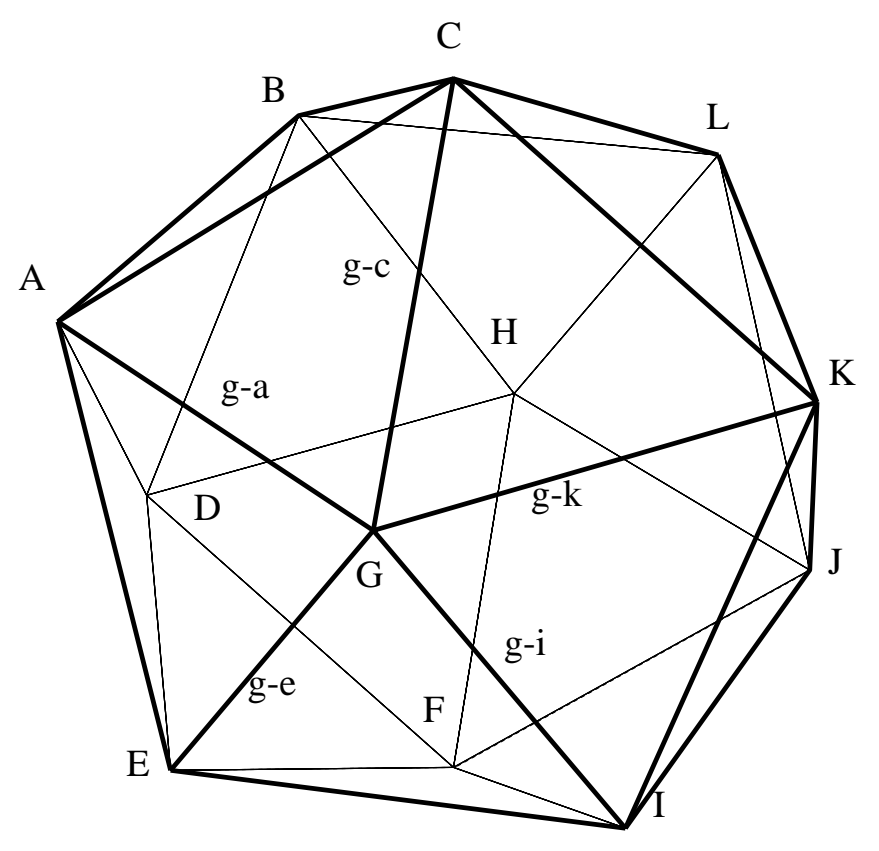

Figure 1. Icosahedron showing the conversational structure of Team Syntegrity

As mentioned before, there is an opening question that focuses the meeting and provides the central topic. The detailed agenda for the discussion is, however, selfbuilt; that is, participants produce it during the event. For this purpose, in a first phase the participants' concerns regarding the opening question are clustered into the twelve main issues or subtopics, to be discussed by the twelve teams (one each). Then the participants indicate which subtopics they would like to discuss the most, and teams are formed according to this criterion. The following phase is devoted to the discussion. For each subtopic there is a number of sessions (usually three) intercalated with those of other subtopics. During each session, all advocates and critics have a certain number of chances to advance their positions or critique what has been said so far, respectively. At the end of each day -in which all subtopics have been discussed once - other mechanisms are used for making the results of the discussions visible to the rest of the participants. 
Extensive use of the methodology has demonstrated that it is particularly useful for supporting teamwork related to planning, innovation and knowledge acquisition processes (Schwaninger, 1997 and 2002); and also for integrating the implicit knowledge and experience in the minds of those working to solve a problem or agree on an important public concern (Pfiffner, 2001; and Stadelman, 2001).

\section{OTHER CONVERSATIONAL APPROACHES IN SYSTEMS THINKING}

Other systems approaches also promote the occurrence of conversation and debate among stakeholders in a situation, as part of democratic processes of problem solving, decision-making, learning, and so on. This is the case of, for instance, Soft Systems Methodology or SSM (Checkland, 1988; Checkland and Scholes, 1990); Strategic Assumption Surfacing and Testing or SAST (Mason and Mitroff, 1981); and Critical Systems Heuristics or CSH (Ulrich, 1987), among others. The democratic nature of all these methodologies differs, however, in terms of the sort of restrictions they impose on the conversation.

Unsurprisingly, all of them have been categorised as mainly belonging to the paradigms called interpretive and emancipatory (Jackson, 2000), given that it is in these paradigms that the existence of different viewpoints is acknowledged. Interpretive systems thinking has been characterised in terms of a number of different aspects, among which are that there is "no assumption that the real world is systemic"; that they do not use models to represent the world, but "to interrogate perceptions of the real world and to structure debate about changes which are feasible and desirable"; and that "the intervention is best conducted on the basis of stakeholder participation" (Jackson, 1999, p.21). SSM and SAST are said to be interpretive approaches. Emancipatory systems approaches would be distinguished from interpretive ones, among other aspects, in that "analysis of the problem situation is designed to reveal who is disadvantaged by current systemic arrangements", "models are used to 'enlighten' the alienated and disadvantaged about their situation and to suggest possible improved arrangements", and in that "the intervention is conducted in such a way that the alienated and/or disadvantaged begin to take responsibility for the process" (ibid.). CSH has been argued to be an example of this type of approach, just like Team Syntegrity (Jackson, 2000).

All the approaches mentioned provide some guidelines for the debate, in order to maximise the learning and understanding that can take place in it. This way, for instance, in SSM the perspectives brought into the conversation are re-described by means of systems ideas, with conceptual tools such as root definitions and the mnemonic CATWOE, to help participants understand and distinguish the various views entering the conversation. It further serves to clarify them as well as some of their implications, in terms of, for instance, what the "system" does (transformation); who is supposed to benefit from it (clients), and what worldview underlies and gives sense to it (weltanschauung). 
Another approach, Ulrich's CSH, similarly provides some guidelines for redescribing the views that enter the conversation, intended in this case to help participants identify the normative and indemonstrable assumptions of each view (Ulrich, 1983 and 1987). The tool used for this purpose is the boundary questions for revealing boundary judgements. Some of these boundary questions refer to similar categories to those specified in SSM, such as clients, purpose, and world-view, while some others are more clearly related to the critical intent behind CSH.

For the present discussion, it is important to notice that these two approaches' rules - or restrictions - for the description of the views that enter the conversation, focus specifically on the contents of those views, and particularly on how those contents should be interpreted. That is, they attempt to give the methodology users some conceptual tools with which they can improve their interpretations of their own as well as other people's views, by identifying some of their assumptions, implications and limitations. This way, for instance, both SSM and CSH remind their users that any purposeful system has certain clients, and that this aspect is crucial when evaluating what action to take. This is of central importance because participants in a conversation may not take that fully into account without a methodology like these that makes that explicit.

For its part, Mason and Mitroff's SAST provides a mixture of restrictions on the contents and on the structure of the conversational interactions (Mason and Mitroff, 1981). About content interpretation, in SAST participants are asked to critically examine all the proposals put forward in the conversation, to determine the assumptions that underlie them, particularly as regards presuppositions about the behaviour of actors who might influence each proposal's success were it implemented. But let us notice that these restrictions provide, just like in the case of SSM and CSH, a way of interpreting or describing the various views - in this case proposed strategies - in a particular way. Restrictions on the structure of interactions in SAST specify how groups for debate will be formed, and when and how participants will be able to discuss their own as well as other groups' proposed strategies for action in the situation being considered. These rules are designed to help in the construction of alternative proposals for action and in their critical questioning, trying to guarantee that all the participants' views will be equally given a chance to be voiced, as well as critically examined.

\section{CRITICAL CONSCIOUSNESS, LEARNING, AND LANGUAGE}

In the previous section we already gave some hints on the issue of how rules of interpretation - or restrictions on content - may affect learning: They direct the methodology users' attention to certain issues which otherwise they might not have considered in their analysis. In order to more thoroughly explain what goes on when restrictions on content are used, we are now going to introduce in this discussion a concept mainly drawn from the pedagogical work of Paulo Freire: critical consciousness (Freire, 1970 and 1973). 
Interestingly, and as has been argued elsewhere (Mejía, 2004), the very concept of critical consciousness necessarily and directly implies a reference to interpretation of contents: A critical person (the person who has developed a critical consciousness) is someone who inquires into ideologies, manifested either in people's ideas and views, or in social reality as constructed by people in a certain way that is deemed critical. Which ways are taken to be critical and which are not, or in other words what kinds of issues, or questions, a critical person is supposed to consider about people's ideas and social reality, can be derived from a review of the theorisation presented by Freire as well as other proponents of critical pedagogy. These are political questions like "what is the ideology that stands behind a particular view or social arrangement?" and "who benefits from the implementation of this ideology in social arrangements or practices?" (Burbules and Berk, 1999). Now, by answering these and similar questions about a particular ideology, one is effectively providing a [critical] description or interpretation of that ideology. Let us now highlight the similarity in the critical role played by Freirean questions about ideologies on the one hand, and by SSM's, CSH's, and SAST's categories for describing or interpreting views or proposed strategies. Both suggest what kinds of questions can help people construct better and more critical interpretations of their own and others' views. One important difference, however, consists in the fact that arguably Freire's pedagogy provides not only the questions, but also strong guidelines as to how those questions should be answered. In this sense, SSM's, CSH's and SAST's restrictions can be called content-empty, whereas Freirean ones are content-full (Mejia, 2002). That is, while the three systems methodologies rely on the participants' critical capacity to identify assumptions by just asking them to do so, Freire's critical pedagogy goes further and guides students (or participants) through a process of becoming critical. Now, it can be argued that during this process, the students learn particular ways of interpreting ideologies and views, that specify the contents of those interpretations (Taylor, 1993; and Mejía, 2004).

The Freirean approach, more radical as concerns restrictions on content in comparison with the other systemic approaches examined before, gives us for that reason a good case for examination. It has been argued elsewhere (Mejía, 2002 and 2004), that the reference to contents that Freire's critical consciousness provides is necessary for becoming an active and critical learner. But why is this so? Why is a guided inquiry into contents needed? We will not reproduce the whole argument here, but it can be summarised as follows: Belief systems or ideologies are not transparent, given, or readily available for questioning; they must be interpreted. Interpretation is a process carried out by some interpreter with the knowledge resources $\mathrm{s} /$ he has available. Because a critical understanding of a belief system requires the use of resources that may lie beyond those presently available to some particular interpreter, $s /$ he might fail to identify and address important issues concerning that belief system. This failure implies that the interpreter simply cannot become totally responsible for the adoption or rejection of the particular ideologies or belief systems under examination, or at least of those aspects of them that $\mathrm{s} / \mathrm{he}$ did not consider. And, importantly for the purpose of this paper, critical learning 
may be impaired. It is crucial to clarify here that, on the other hand, a methodology that compels its users to reflect on specific issues, does not guarantee that all relevant aspects will be reflected on either. It is only guaranteed that they will reflect on the specific issues the approach explicitly mentions, which of course may not cover everything that is important or relevant.

The systemic methodologies mentioned above will in essence do the same as a radical approach such as Freire's, given that they are also based on interpretative restrictions on content. However, there is a difference in that a more radical and fully-fleshed approach like Freire's will specify to a greater degree the contents that it introduces for reflection and discussion. This has at least two consequences: The first one is that it is made sure with more certainty that specific issues will be reflected on or discussed. In general, the more specified the contents introduced by the methodology, the more it is made sure that the issues involved in those contents will be reflected on and discussed. The second consequence refers to the fact that the introduction of more specific content implies that there is more knowledge, or beliefs, that the methodology users will have to adopt in order to develop the critical consciousness that allows them to produce critical interpretations of views or proposals for action. And this in turn means that they will be more prone to having that knowledge imposed on them. This tension, however, seems an insoluble consequence of language. A more detailed treatment of these issues appears elsewhere (Mejía, 2002).

\section{CONVERSATIONAL RESTRICTIONS AND TEAM SYNTEGRITY}

This argument is connected, precisely, with the way we are proposing to understand how conversational rules or restrictions may affect learning. We have already distinguished between those rules or restrictions on the content of the belief systems that enter the conversation, or their interpretations, and those on the structure of the interactions among the participants in conversation.

From our description of the protocol in section 1, however, it can be seen that Team Syntegrity does not provide content-based restrictions. That is, there are no rules that in a direct way mention anything in particular about contents, except for the agreement to meet and discuss on a particular opening question. There are no specific aspects suggested to be thought of or discussed, and no guides as to how to interpret the ideas entering the conversation. The protocol focuses on the form that the interaction structure should take if there is to be maximum constructive engagement of the views brought in by the participants. It tries to make sure, for instance, that no single person or group will dominate the conversation by being the ones who talk, while the others only listen. Additionally, it tries to make sure that what is said by any participant will reverberate throughout the whole arrangement of discussants - even in the teams s/he does not belong to-and that therefore it is more likely that it will be taken seriously in the conversation. But these restrictions say nothing about content in any direct way, and thus one might conclude that it 
cannot be guaranteed that the infoset will consider some particular important issues, or reflect on important taken-for-granted assumptions. However, this conclusion may have been arrived at just too quickly: one may still ask whether, even if only indirectly, these structure-based restrictions will have some impact on the conversational contents. In what follows we will examine some particular elements of the protocol that we think may have some such impact, and explore how that impact may depend on some additional clarification in the roles involved in a Team Syntegrity event.

The participant role. Given that Team Syntegrity does not deal with contents, it is up to the participants what topics, aspects, or perspectives are considered or discussed. But, of course, different participants will bring different perspectives into the conversation. The actual conversational contents will therefore correspond, at least to some extent, to the participant selection. If the selection excludes some particular social actors, relevant regarding the opening question, then it is likely that some important perspectives will be excluded as well. This problem can be solved by simply maximising the variety of backgrounds and ideological orientations of the participants, so that the content resources entering the conversation are maximised as well. This is certainly a criterion that has been used before (Espinosa, 2003). Nevertheless, this solution can sometimes be impossible to apply to a full extent, due to difficulties ranging from financial and logistic ones - e.g. bringing persons to the physical location of the event or restrictions in the number of participants - to others such as the impossibility of some to participate in this or any conversation due to their nature or present state-e.g. the environment, the dead, the not-yet-born, the not-yet able to participate in conversations of this type, the mentally handicapped, the animals, etc. - (Pearson, 1994). Additionally, some may not be able to properly represent their own interests, for instance because they are in a state of alienation or false consciousness (Freire, 1970; Midgley, 1997). More generally, this concern suggests the possibility that a participant might lack autonomy and not behave as a critical learner, due to individual or social learning impediments. To summarise, the best representation of stakeholders will make the best option of having a truly democratic context, but it will only be fully used if participants are autonomous active learners (Espinosa, 2000).

The critic role. As described in section 1, critics have the devil's advocate role. They point out the possible ways in which the conversation by the discussants may not be going well, or may be lacking in some respect. This may include revealing presuppositions not properly examined, or aspects or dimensions not taken into consideration. This role is particularly important for our discussion in this paper: In terms of the terminology we have introduced, one of their tasks is to bring into the conversation those contents that are relevant but that the discussants are not dealing with, or to re-address the ways in which contents are currently being discussed. Referring now to our discussion about participant selection, if the critics play their role properly, exclusions in the selection process might be compensated for by means of the critics' introduction of contents that correspond to the perspectives of others who are not participating in the event. At this level, we would like to leave open the 
question as to how likely it is that critics will make a conscious sufficient effort to step into the shoes of those who are not present, or are incapable of properly representing their own interests.

The facilitator role. From the organising team, the facilitators are direct witnesses of the discussions that occur within the teams, and have a certain authority for making the discussants stick to the conversational rules specified by the protocol. Their task, as indicated by their role name, is simply to help the discussion flow by means of some regulating actions (like turn assigning) and other forms of support such as writing the ideas stated by both discussants and critics. In contrast, a critical facilitator would have the responsibility for becoming aware of exclusions in the discussions within the teams. They could then try to compensate in some way for those exclusions, whenever possible, by means of either a direct inclusion of new ideas about unquestioned presuppositions, unexamined dimensions, and so on, or by means of the asking of questions that can help participants do it (Gregory and Romm, 2001). (These two different options correspond to the distinction made in section 3 of this paper, between radical and fully-fleshed approaches such as Freire's on the one hand, and those which only establish some generic categories for reflection, such as SSM and CSH.) A critical facilitation would also be alert to the presence of subtler mechanisms of exclusion that could appear despite the protocol's intention to prevent it. One such possible and not uncommon mechanism is simply ignoring what a person says, by not engaging with the ideas or dimensions s/he brings into the conversation. Another possible mechanism is an unequal distribution of the burden of proof in the conversation; that is, that during a conversation there is pressure on the advocates of some position to strongly argue why they support it, whereas the advocates of an alternative position are not demanded to do so. Presently, it is the task of those in the role of critics within each discussion, to take responsibility for pointing out those exclusions; but as the discussion about the critic role suggests, it might difficult to guarantee that it will occur.

Surely enough, if it is intended to promote critical active learning in the participants, it is our contention that some of the limitations described can be dealt with by adding or modifying particular elements in the Team Syntegrity protocol. It is not within the scope of this paper, however, to make suggestions as to how to do that; that should indeed be the subject of further research. Some other limitations may nevertheless correspond to unsolvable issues, about which one can only adopt a purely negative critical position: to make them explicit, as well as their possible consequences, and to assume responsibility (Ulrich, 1983 and 1987).

\section{FINAL REFLECTIONS}

It seems clear to us, mostly from our own experience in using Team Syntegrity as a tool for developing democratic contexts, that it has significant potential. This paper introduced new questions that address issues that in the future might help improve this potential, focusing on how to better enhance individual as well as group critical 
learning. We must learn to learn critically, both individually and as organised social groups, accepting our restrictions as learners but using the best of our own current expertise as a resource for an improved learning. More questions coming from our arguments also need further research, like those referring to the required skills for engaging in a conversation and participating effectively, under individual, social, cultural and political restrictions.

In the light of this, our discussion can be seen as an attempt to contribute to give some structure to current academic debate around these issues, as a way to understanding further the possibilities and limitations of systemic conversational protocols, regarding the development of critical active learners. The analysis of Team Syntegrity showed the difference between structure and content based conversational restrictions, in terms of their plausible effects on individual and group learning. It also highlighted some aspects of the protocol which, if developed, could help improve the evolvement of critical consciousness. More research is required, however, in order to on the one hand refine the conceptual conclusions about language, learning, and critique, and on the other to develop and test proposals, based on this analysis, for improving various conversational tools, such as Team Syntegrity. We hope we somehow opened ways for this kind of research to continue in order to get deeper results and findings.

\section{ACKNOWLEDGEMENTS}

We would like to thank Professor Mike C. Jackson at Hull University, as well as Joe Trust and Chris Cullen at Team Syntegrity Inc., for their helpful comments and clarifications on previous versions of this paper.

\section{REFERENCES}

Beer, S. (1994). Beyond Dispute. The invention of Team Syntegrity. Wiley, Chichester. Brady, J. (1994). Critical Literacy, Feminism, and a Politics of Representation. In Politics of liberation: Paths from Freire (P. McLaren and C. Lankshear, eds.). Routledge, London, 142-153.

Burbules, N., and Berk, R. (1999). Critical Thinking and Critical Pedagogy: Relations, Differences, and Limits. In Critical Theories in Education: Changing Terrains of Knowledge and Politics (T. Popkewitz and L. Fendler, eds.). Routledge, New York, 4565.

Checkland, P. (1988). Soft Systems Methodology: An Overview. Review of Applied Systems Analysis, vol 15.

Checkland, P., and Scholes, J. (1990). Soft Systems Methodology in Action. Wiley, Chichester.

Espinosa, A. (2000). Creating A Democratic Learning Context: An experience in the use of Syntegrity for this purpose, Proceedings of the World Congress on the Systems Sciences and ISSS, Toronto, July 2000. 
Espinosa, A. (2003). Team Syntegrity as a Tool to Promote Democratic Agreements: An Example from the National Environmental Sector in Colombia, Proceedings of the ISSS -2003 Conference Agoras in the Global Village, Crete, July 2003.

Freire, P. (1970). Pedagogy of the Oppressed. Seabury, New York.

Freire, P. (1973). Education for Critical Consciousness. Seabury, New York.

Gregory, W. and Romm, N. (2001).Critical Facilitation: Learning Through Intervention in Group Processes. Management Learning, vol.32, no.4, 453-467.

Jackson, M. (1999). Towards Coherent Pluralism in Management Science. Journal of the Operational Research Society, vol.50, no.1, 12-21.

Jackson, M. (2000). Systems Approaches to Management. Kluwer, New York.

Jackson, M. (2003) Systems Thinking: Creative Holism for Managers, Wiley, Chichester.

Mason, R. and Mitroff, I. (1981). Challenging Strategic Planning Assumptions: Theory, Cases, and Techniques. Wiley, New York.

Mejía, A. (2002). Can the System Idea Help Promote Critical Thinking and Intellectual Autonomy in Pedagogy? Kybernetes, vol.30, nos.9/10, 1313-1323.

Mejía, A. (2004). The Problem of Knowledge Imposition: Paulo Freire and Critical Systems Thinking. Systems Research and Behavioral Science, vol.21, no.1, 63-82.

Midgley, G. (1997). Dealing with Coercion: Critical Systems Heuristics and Beyond. Systems Practice, vol.10, no.1, 37-57.

Pearson, A. (1994). You Drive for Show but You Put for Dough: A Facilitator's Perspective. In Beyond Dispute: The Invention of Team Syntegrity (S. Beer). Wiley, Chichester, 313-322.

Pfiffner P. (2001). Team Syntegrity - Using Cybernetics for Opinion Forming in Organisations. In M.o.M. Malik on Management, Nr. 5/01.

Stadelman, P. (2001). Team Syntegrity: Scientifically based way for integrating the distributed knowledge to find solutions. In M.o.M Malik on Management. Nr. 5/01.

Schwaninger, M. (1997). Self-Organization and Self-Reference in the Cognition of Organizations: In Interdisciplinary Approaches to a New Understanding of Cognition and Consciousness (Braitenberdg, V., Radermacher, F. and Josef, eds.). Unipress 03-1997, Die Zoltscrhrilft des Universitat Augsburg.

Schwaninger,M. (2002). Enhancing Organisational Cognition. SEM/Radar, Systemdenken und Entscheldungsfinung im Management, 01, 2002.

Taylor, P. (1993). The Texts of Paulo Freire. Open University, Buckingham.

Ulrich, W. (1983). Critical Heuristics of Social Planning: A New Approach to Practical Philosophy. Wiley, Chichester.

Ulrich, W. (1987). Critical Heuristics of Social Systems Design. In Critical Systems Thinking: Directed Readings (R. Flood and M. Jackson, eds.). Wiley, Chichester, 103115, 1991. 